\title{
Cyberphysical Network Applied to Fertigation Agricultural Processes ${ }^{+}$
}

\author{
Higor Vendramini Rosse ${ }^{1, *, \ddagger(\mathbb{D})}$ and João Paulo Coelho ${ }^{1,2, *, \ddagger(\mathbb{D})}$ \\ 1 Instituto Politécnico de Bragança, Campus de Santa Apolónia, 5301-857 Bragança, Portugal \\ 2 Centro de Investigação em Digitalização e Robótica Inteligente (CeDRI), Campus de Santa Apolónia, \\ 5300-253 Bragança, Portugal \\ * Correspondence: higorvendraminirosse@gmail.com (H.V.R.); jpcoelho@ipb.pt (J.P.C.) \\ + Presented at the 2nd XoveTIC conference, A Coruña, 5-6 September 2019. \\ $\ddagger$ These authors contributed equally to this work.
}

Published: 31 July 2019

\begin{abstract}
Fertigation is a widely used crop growing method that consists on the precise injection of a nutrient solution that commonly consists on a mixture of three basis components (nitrogen, phosphorus and potassium) diluted in water. This nutritive suspension is delivered to the plants with a frequency and relative basis contents that depends on the plant's type, its vegetative state and actual environmental conditions. This production process is becoming increasingly popular due to several advantages over more traditional approaches such as more control on the plant fertilisers and an increasing reduction of irrigation water. This is achieved by an increase complexity on the crop growing process management which requires a technological layer responsible for mixing the nutrients and monitoring the local environmental conditions. Despite this technical component, the short and long term management decisions depend almost exclusively on the grower's experience and intuition. This type of human-on-the-loop control can lead to a suboptimal use of resources wish can translate into reduction of economic profit and an can lead to waste of water and fertilisers. In this context, decision support mechanisms based on artificial intelligence and machine learning algorithms can be of extreme relevance in order to steer the grower decisions and increase the overall production process efficiency. The performance of those types of approaches strongly relies on the availability of data which can be both local and global. This work deals with the architecture of a sensor network which will be responsible to gather local information on the actual growing conditions. Those conditions are usually not homogeneous within the complete production plant and must be taken into consideration. In particular, the current architecture vision will consider those clusters, where the environmental conditions are similar, as cyberphysical devices. These devices will consist on vegetative production area, sensor networks and local control of irrigation state.
\end{abstract}

Keywords: agriculture; fertigation; cyberphysical devices; sensor network

\section{Introduction}

Fertigation is a cultivation technique that relies on the precise injection of the nutrient solution according to plant requirements, environmental and soil/substrate conditions. The use of fertigation systems has become popular as an alternative to more classical cultivation techniques. Indeed, more than 11 million hectares of area are associated with fertigation-based production schemes. This mark tends to increase due to the advantages promoted by this method, among which are the reduction of fertilisers, phytopharmaceuticals and water consumption. However, these advantages are achieved at the expense of rigorous monitoring of the nutrient solution, environmental conditions and vegetative state of the crop. Maintaining rigid management of these conditions is a difficult process due to several 
factors: first, the high area of cultivation normally at stake requires a large number of human resources to achieve the necessary monitoring accuracy and second, the information on the current crop state is taken on average, even if there is heterogeneity in the vegetative state of some plant clusters within the universe of the total production. For these reasons, complete automation of the irrigation process should be considered in order to characterise, in real-time, the actual state of the crops and promote the adjustment of nutrient according to fluctuations in environmental conditions and low frequency deviations due to vegetative evolution of the crop. Thus, it is proposed the incorporation of artificial intelligence and machine learning mechanisms merged with cyberphysical units as a way to create a decision support system.

This approach considers the fertigation crop growing system equivalent to an industrial production line where the raw material enters, a set of operations is performed and the final product is created. In this case, the mixture of fertigation, solar radiation and relevant environmental variables are the raw input material. The photosynthesis and other biological functions are compared to the operations performed and the vegetable matter generated the final output. Due to the similarity between common industrial processes and the production scheme based on fertigation, it makes sense to migrate some of the concepts presented in the Industrie 4.0 paradigm to this agricultural production reality. In particular, those that lead to an increase in robustness, adaptability, lean and waste reduction. Among other approaches, the use of decentralised agent-based control methods has proven to be an efficient solution in the management of complex industrial systems. Those agents will responsible to locally manage each cyberphisical cluster. Each cyberphysical elements is composed of areas of vegetative production, sensor networks and local control of the state of irrigation. In a typical fertigation production system, there will be a large number of cyberphysical elements that must be scaled up or down according to the production schedule. Notice that, although each cyberphysical element is considered to have local processing capacity, these will be vertices in an integrated system that will take place on a cloud computing platform (FIWIRE). This work deals with the cyberphisical element architecture which will be present in the next section.

\section{The Cyberphysical Ecosystem}

The full crop production area will be divided into cyberphysical elements. Each cyberphysical element will be composed by a set of neighbour crop growing lines that share common environmental conditions. A set of measurement nodes (MOTES) will be distributed along the growing stands gathering information on substrate moisture, air temperature, solar radiation, $\mathrm{pH}$, electrical conductivity, nitrites and nitrates. Artificial vision is also being considered in order to estimate the vegetative state of the crop. But at this point, the sensor network only involves data from environmental and substrate conditions. The overall architecture of one of such cyberphysical elements is presented in Figure 1.

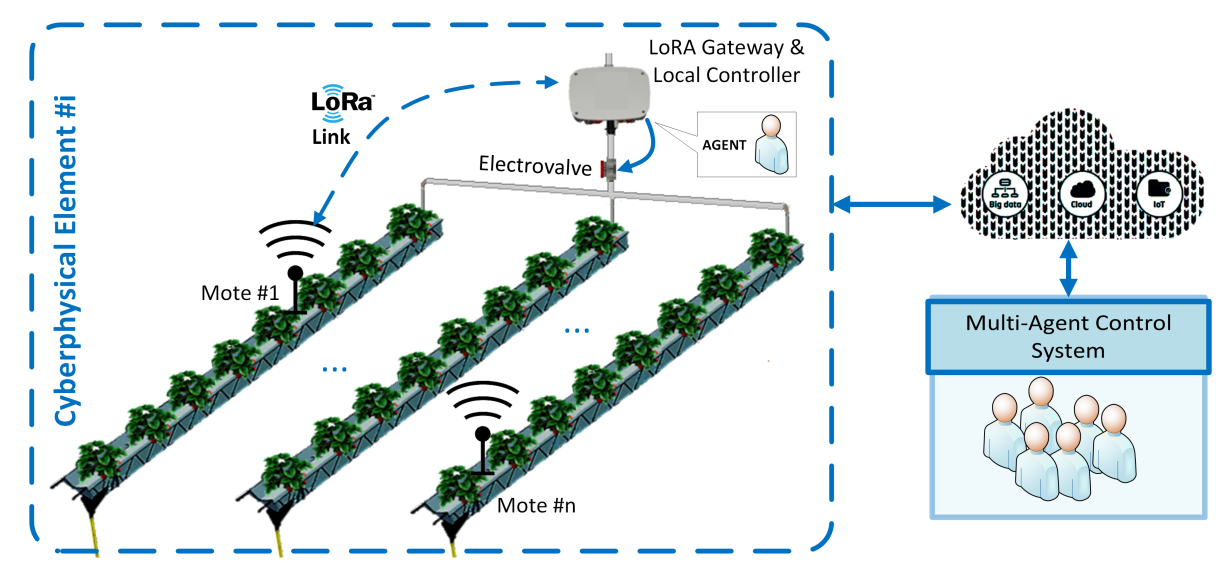

Figure 1. Schematic diagram of the cyberphysical fertigation element architecture. 
Each MOTE consists of a local low power processor, a set of sensors such as temperature, solar radiation, soil moisture and water level, and a transceiver. The information sent by the MOTES will be filtered and organised by a software agent which is also responsible to communicate with the FIWIRE platform. The communication between the virtual agent and the motes is carried out through a Gateway connected to the motes by using a wireless point-to-multipoint LoRAWAN based network architecture. Whenever required, the agent sends a message to the MOTES requesting the sensors information. At the present time, we are engaged in developing the MOTES hardware and firmware and evaluate the sensors technologies to be used.

Author Contributions: The original idea and concept was put forward by J.P.C and both the methodology and paper writing were carried out by H.V.R and J.P.C.

Funding: This research received no external funding.

Conflicts of Interest: The authors declare no conflict of interest.

(C) 2019 by the authors. Licensee MDPI, Basel, Switzerland. This article is an open access article distributed under the terms and conditions of the Creative Commons Attribution (CC BY) license (http:/ / creativecommons.org/licenses/by/4.0/). 